\title{
Verbal encouragement: effects on maximum effort voluntary muscle action
}

\author{
Peter J McNair, Jill Depledge, Matt Brettkelly, Stephen N Stanley
}

\begin{abstract}
Objective-To examine the effects of verbal encouragement on the peak force of the elbow flexors during an isometric muscle action.

Methods-A crossover design whereby 20 subjects were divided into $102 \times 2$ Latin squares was undertaken. Peak forces were measured on a Kin-Com dynamometer, and electromyographic (EMG) activity was also recorded from the biceps brachii. All subjects completed trials with and without verbal encouragement.

Results-Mean peak force increased $(P<0.05)$ from 296 to $311 \mathrm{~N}(5 \%)$ when verbal encouragement was presented. A spectral analysis of the EMG activity showed no changes $(P>0.05)$ to the median frequency in the condition where verbal encouragement was present.

Conclusions-These findings have ramifications for training and exercise therapy. An awareness of the effects of verbal encouragement is important when motivating athletes and patients to attain maximum performance during exercise. (Br $\mathcal{F}$ Sports Med 1996;30:243-245)
\end{abstract}

Key terms: strength; verbal; performance; psychological influences

The development of muscle tension involves both neural and morphological factors. The neural factor involves the recruitment of motor units and the modulation of their firing rates. ${ }^{1}$ To generate maximum force in a muscle, an individual must recruit all of the muscle's fibres at their maximum firing rate. ${ }^{1}$ In some individuals, there is evidence that during a maximum voluntary contraction, the activation of the muscle fibres may be inhibited.' This inhibition has been linked to supraspinal "drive" acting on the motor units. ${ }^{1}$ In this respect, the idea and motivation for generating maximum muscle tension evolves in the brain and involves the limbic system and cerebral cortex. ${ }^{2}$ There is evidence that unusually stressful situations, for instance seeing a person trapped under an object, or specific sensory stimuli such as a sudden loud noise may disengage this supraspinal inhibition and lead to an enhancement of strength. ${ }^{3}$ In regard to the latter scenario, pathways have been identified between the components of the auditory system and the motor system (for example, the startle reflex). ${ }^{4}$ However, there is conflicting evidence that verbal commentary can lead to improvements in strength. ${ }^{56}$ Hence, the purpose of the current study was to examine the effects of verbal encouragement on the peak force generated by the elbow flexors and the associated EMG activity from these muscles during maximum isometric muscle action.

\section{Methods}

SUBJECTS

Twenty healthy subjects (10 male, 10 female) with a mean age of 22 years (range 20 to 28 years) volunteered to participate in this study. None was a trained athlete or undergoing strength training programmes. Informed consent was obtained from all subjects. A crossover design was undertaken, and subjects were paired by gender, height, and weight to form $102 \times 2$ Latin squares. Within each square, subject 1 received non-verbal encouragement followed by verbal encouragement and subject 2 received the reverse order.

\section{STRENGTH TESTING}

Subjects were positioned sitting and stabilised by straps on a Kin-Com dynamometer, with the right shoulder in the anatomically neutral position and the elbow flexed to 90 degrees. The shaft of the dynamometer was aligned with the approximate rotational axis of the elbow. After a warm up of submaximal exercise, subjects performed two sets of three maximum effort isometric contractions of the elbow flexors in this position. The duration of each contraction was $5 \mathrm{~s}$ and a 1 min rest interval was provided between each maximum effort. In the set of contractions where verbal encouragement was provided, the words spoken were "Come on, you can do it", and they were repeated for the duration of the contraction. The words were spoken by the same researcher to all subjects at a volume slightly louder than that occurring during conversation. The highest peak force recorded across the three trials in each condition was used in the analyses of these data.

\section{ELECTROMYOGRAPHY}

Electromyographic (EMG) activity from the biceps brachii was chosen to be representative of the muscle activity from the elbow flexors. Following appropriate skin preparation, surface electrodes (3M:2259) were placed over the muscle belly. The signals were amplified $(\times 2000)$, band passed ( $5 \mathrm{~dB}$ down at $3 \mathrm{~Hz}$ and $1 \mathrm{kHz}$ ) and relayed to a computer based data acquisition system operating at a sampling frequency of $1000 \mathrm{~Hz}$. The trial in which the peak force was recorded was used in the analysis of 
Mean (SEM) peak force and median frequency recorded with and without verbal encouragement.

\begin{tabular}{lll}
\hline & Force (N) & Frequency (Hz) \\
\hline Verbal & $311(23)$ & $79.0(2.5)$ \\
Non verbal & $296(22)$ & $79.0(2.7)$ \\
\hline
\end{tabular}

the electromyography data. This signal was detrended with a least squares calculation and a trapezium taper was then applied. The power spectra could then be derived using a Fast Fourier transformation. The median frequency was obtained by summing the power estimates at incremental frequencies to obtain a cumulative power. The frequency at which half the cumulative power occurred was defined as the median frequency.

\section{STATISTICAL ANALYSIS}

The dependant variables were the peak force and median frequency generated in each condition. The $t$ distribution was used to establish any statistical differences in the force and EMG data. The effects of gender were examined using the percent difference between the verbal and non-verbal trials. An additional test to establish if an order effect was apparent was also undertaken. ${ }^{7}$ The $\alpha$ level was set to 0.05 for these tests.

\section{Results}

The table presents the results for peak force recorded with and without verbal encouragement. There was a significant increase $(P<0.05)$ in the peak torque recorded when verbal encouragement was present. The magnitude of this increase was a mean of $5 \%$. No significant gender effect was observed $(P>0.05)$. The table also shows the results for the median frequency. No significant difference was observed in the median frequency between the verbal and non-verbal conditions. A test to determine whether a "carry over" effect from one condition to the other existed showed there was no statistical evidence $(P>0.05)$ for this effect occurring.

\section{Discussion}

In the current study, a $5 \%$ increase in peak force was observed when subjects were verbally encouraged. In regard to verbal communication, it is apparent that the amplitude and the choice of words are important factors Johannsson et $a l^{6}$ noted an $8 \%$ increase in strength when the amplitude of verbal instruction was increased by $22 \%$. These findings may be related to those of other researchers ${ }^{3}$ who noted increased strength in individuals who had been subject to a loud noise such as gunfire at close range.

In the study by Johannson et al study, the verbal instructions contained no words of encouragement, which is in contrast to our study, where the words used were "encouraging" the subject to generate more force. Ikai and Steinhaus ${ }^{3}$ have shown that hypnotic suggestions for being "stronger" and "weaker" can increase and decrease an individual's abil- ity to generate maximum strength by $20-30 \%$. Later work ${ }^{89}$ has also shown that techniques such as self efficacy statements, visualisation, and imagery can influence the performance of individuals in strength and power activities. These findings suggest that such strategies focus the subject's attention on the task to be performed. ${ }^{8}$ In contrast to our findings were those of Rube and Secher, ${ }^{5}$ who noted that "cheering" subjects had no effect on strength values.

The power spectral analysis of EMG activity has been used to study the activation of muscle. ${ }^{10}$ In such studies, changes in the median and mean frequencies have been observed. Mathematical models ${ }^{11}$ and direct measurement of muscle fibre conduction ${ }^{12}$ support the view that shifts in the power spectrum are associated with the conduction velocity of the muscles fibres. Given that higher conduction velocities are observed in fast twitch fibres which are normally activated in maximum effort contractions, ${ }^{13}$ it was thought that enhancements of strength as a result of verbal encouragement might be reflected in a greater median frequency of the activated muscles, thus showing enhanced activation of type 2 fibres. However, no change in the EMG activity was observed in the current study. It may be that the median frequency is more sensitive to changes in recruitment than firing rate, ${ }^{14}$, and there is evidence ${ }^{15}$ that recruitment of motor units in the biceps brachii would be complete by $80 \%$ of a maximum voluntary contraction. Alternatively, changes in the median frequency may have occurred in the major elbow flexor, brachialis, the activity of which cannot be recorded by surface EMG.

\section{CONCLUSIONS}

Motivating individuals is an essential aspect of training and exercise therapy. It is important that coaches and clinicians have an awareness of the potential for athletes and patients to increase their performance through appropriate verbal encouragement. The results of this study indicate that words of encouragement spoken at a slightly raised volume can lead to small but significant increases in strength within a single session.

1 Belanger AY, McComas AJ. Extent of motor unit activation during effort. ₹ Appl Physiol 1981;51:1131--5.

2 Enoka R. Neuromechanical basis of kinesiology. Champaign: Human Kinetics, 1988:183--211.

3 Ikai M, Steinhaus A. Some factors modifying the expression of human strength. $\mathcal{F}$ Appl Physiol 1961;16:157--63.

4 Rossignol S. Startle responses recorded in the leg of man. Rossignol S. Startle responses recorded in the leg of
Electroencephalogr Clin Neurophysiol 1975;39:389--97.

5 Rube N, Secher NH. Paradoxical influence of encourage-

Rube N, Secher NH. Paradoxical influence of encourage-
ment on muscle fatigue. Eur $\mathcal{f}$ Appl Physiol 1981;46:1--7.
Johansson CA, Kent BE, Shepard KF. Relationship between Johansson CA, Kent BE, Shepard KF. Relationship between
verbal command volume and magnitude of muscle contraction. Phys Ther 1983;63:1260--5.

7 Fleiss J. The design and analysis of clinical experiments. New York: John Wiley and Sons, 1986:263--90.

8 Wilkes R, Summers J. Cognitions, mediating variables, and strength performance. $\mathcal{F}$ Sport Psychol 1984;6:351--9.

9 Tenenbaum G, Bar-Eli M, Hoffman J, Jablonovski R, Sade $S$, Shitrit D. The effect of cognitive and somatic psyching-up techniques on isokinetic leg strength. $f$ Strength Conditioning 1995;9(1):3-7.

10 Gerdle B, Eriksson N, Brundin L. The behaviour of the mean power frequency of the surface electromyogram in the biceps brachii with increasing force and during fatigue. Electromyogr Clin Neurophysiol 1990;30:483--9. 
11 Stulen F, De Luca C. Frequency parameters of the myoelectric signal as a measure of muscle conduction myoelectric signal as a measure of muscle cond
velocity. IEEE Trans Biomed Eng 1981;28:515--23.

12 Arendt-Nielson L, Mills K. The relationship between mean power frequency of the EMG spectrum and muscle fibre power frequency of the EMG spectrum and muscle fibre
conduction velocity. Electroencephalogr Clin Neurophysiol conduction velocit

13 De Luca C. Control properties of motor units. $\mathcal{F}$ Exp Biol 1985;115:125--36.

\section{Commentary}

This interesting paper attempts to address a logical question of whether strength and EMG activity is mediated by verbal encouragement in a gross motor task. Although the results support the use of verbal encouragement, I have some reservations about the statistical methods adopted. It is disappointing that such a well designed study fails to explore the full potential of the question. The analysis ignores the potential for a gender by treatment interaction. Independent groups $t$ tests systematically collapse the data across each independent variable. A gender by condition analysis of variance would have been preferable and the most appropriate statistical method to analyse the EMG and the strength data. In this way, the potential interaction of gender and condition could have been explored

The authors correctly conclude that verbal encouragement has the potential to improve performance. This is one of the strategies that a sports psychologist could advocate in mental training programme. Researchers interested in such psychological strategies may also wish to refer to such journals as fournal of Sports Psychology, The Sports Psychologist, fournal of Sport and Exercise Psychology, International fournal of Sports Psychology, and Perceptual and Motor Skills among others.

The authors acknowledge that an increased EMG signal was expected in the verbal condition. The explanation for its absence is plausible, particularly as the brachialis is the primary flexor of the elbow and its activity would not be directly measurable by surface EMG, due to its location. A study which uses needle EMG would perhaps enable the question to be answered more fully. In summary, the conclusions of the study, although pertinent, are limited by methodological constraints.

Division of Health and Human Performance,

ROGER G ESTON 